\title{
Nerve Terminal Growth Remodels Neuromuscular Synapses in Mice following Regeneration of the Postsynaptic Muscle Fiber
}

\author{
Yue Li and Wesley J. Thompson \\ Section of Molecular Cell and Developmental Biology, School of Biological Sciences, and Institutes for Cell and Molecular Biology and Neuroscience, \\ University of Texas at Austin, Austin, Texas 78712
}

Muscle fibers degenerate and regenerate in response to contractile damage, during aging, and in various muscle diseases that weaken the fibers. It is known that degeneration and regeneration of the segment of the postsynaptic fiber produces dramatic alterations in the neuromuscular junction (NMJ) that forms on the regenerated fiber, but the mechanisms here are incompletely understood. We have used a laser microbeam to damage the postsynaptic fibers at individual NMJs in the sternomastoid muscle of living young adult mice and then followed the synapses vitally over time using fluorescent proteins expressed in motor neurons and glial cells and staining of postsynaptic acetylcholine receptors. We find, in contrast to previous reports, that the mouse nerve terminal retains contact with the synaptic basal lamina marked by cholinesterase staining even in the absence of the target, showing that this terminal does not require a continuous supply of target-derived molecules for its maintenance. Thus, remodeling of the nerve terminal during the period of target absence does not explain the subsequent changes in the new NMJ. Rather, we see that the synapse becomes altered as the new fiber segment regenerates. Mechanisms for remodeling the synapse include failure of the regenerating muscle fiber to contact the old basal lamina and nerve terminal, growth of the nerve terminal and its glia toward the regenerating fiber, and remodeling of the initial contact as the nerve terminal becomes varicose.

\section{Introduction}

Synapses differentiate and are maintained via reciprocal interactions between nerve terminals and targets. Studies of the neuromuscular junction (NMJ) illustrating these interactions include ablation of muscle fibers (Duchen et al., 1974; McMahan and Slater, 1984; Yao, 1988; Rich and Lichtman, 1989a). A number of trophic interactions have been identified (Sanes and Lichtman, 2001). Surprisingly, in the frog, deletion of the postsynaptic muscle fiber does not change the gross morphology of the nerve terminal (Yao, 1988; Dunaevsky and Connor, 1995). These terminals remain intact and apposed to the specialized portion of the basal lamina present between the nerve and muscle fiber, the synaptic basal lamina, even though Schwann cells (SCs) come to wrap them (Yao, 1988). They even continue to release neurotransmitter upon stimulation (Dunaevsky and Connor, 1998). This contrasts with the situation in the mouse, where fiber abla-

Received June 11, 2011; revised July 20, 2011; accepted July 25, 2011.

Author contributions: Y.L. and W.J.T. designed research; Y.L. and W.J.T. performed research; Y.L. analyzed data; Y.L. and W.J.T. wrote the paper.

This work was supported by National Institutes of Health Grant NS 20480. Confocal microscopy was performed at the University of Texas Institute of Cell and Molecular Biology Core Facility. We thank Yi Zuo for help with microelectrode experiments, Rick Rotundo for guidance in preparing fluorochrome-conjugated fasciculin, Mendell Rimer for a gift of antibody, Jon Pierce-Shimomura for loan of essential equipment, and Young il Lee and Michelle Mikesh for discussions.

The authors declare no competing financial interests.

Correspondence should be addressed to Wesley J. Thompson, 1 University Station, University of Texas at Austin C1000, Austin, TX 78712.E-mail:wes@mail.utexas.edu.

DOI:10.1523/JNEUROSCI.2953-11.2011

Copyright $\odot 2011$ the authors $\quad 0270-6474 / 11 / 3113191-13 \$ 15.00 / 0$ tion is reported to cause rapid remodeling of the nerve terminal (Rich and Lichtman, 1989a). The differences between rodent and frog have been attributed to the degree of reliance upon the postsynaptic target for synaptic maintenance. In the experiments in the mouse, in contrast to those in the frog, the muscle satellite cells were not destroyed by $\mathrm{x}$-irradiation, so new myoblasts quickly repair the fiber damage, regenerating the damaged fiber segment. This regenerated fiber is quickly reinnervated by the nerve terminal, but the regenerated synapse achieves a new, distinct morphology (Rich and Lichtman, 1989a). This is a clear example of plasticity of a synapse that normally is very stable (Magrassi et al., 1987), even during growth and shrinkage of muscle fibers (Balice-Gordon et al., 1990). However, the mechanisms creating this synaptic instability and the extent to which they involve changes occurring during the period of target deprivation are unclear. This is an important issue not only because it addresses questions of synapse maintenance but also because events that damage muscle fiber segments, including those present at the synapse, are more common than generally recognized. They occur in response to muscle contractions during lengthening (i.e., eccentric) contractions (Brooks and Faulkner, 1990), in muscle diseases including several of the muscular dystrophies (Lyons and Slater, 1991), and at high frequency in aging muscle (Valdez et al., 2010).

Here, we report the results of experiments in which we have ablated the segment of the muscle fiber underneath NMJs using a laser microbeam. We find that the nerve terminal remains intact during the period in which the target degenerates but begins to 
change as the target regenerates. These changes are due to induction of growth of the nerve terminal and its Schwann cells. New contact sites are formed as this growth extends toward the regenerating fiber both beneath and to the sides of the previous synaptic site. Additional portions of nerve terminals never become synaptic because they and their associated basal lamina never contact the regenerating fiber. Thus, the remodeling of synapses that occurs on injured muscle fibers is explained by growth rather than by loss of terminal branches occurring as a result of loss of the target.

\section{Materials and Methods}

Animals. Mice studied in these experiments were commonly C57BL/6 of both sexes. Some of the transgenic lines had been prepared in a hybrid C57BL/6DBA mouse but were commonly backcrossed to C57BL/6. Two separate transgenic lines were used: one was a double-transgenic mouse with transgenes coding for expression of cytoplasmic CFP in motor axons [driven by the Thyl promoter (Feng et al., 2000)] and cytoplasmic EGFP in Schwann cells [driven by the S100 promoter (Kang et al., 2003)]. A second transgenic line (line $\mathrm{YH}$ ) expressing cytoplasmic YFP under the S100 promoter (Zuo et al., 2004) and labeling macrophages and immune cells was used in a set of experiments. All experiments were approved by the University of Texas Institutional Animal Care and Use Committee.

In vivo imaging. Vital imaging was conducted following published methods (Lichtman et al., 1987). Briefly, mice were anesthetized by intraperitoneal injection of ketamine/xylazine $(0.10-0.15 \mathrm{ml}$ of a $0.9 \%$ $\mathrm{NaCl}$ solution containing $17.4 \mathrm{mg} / \mathrm{ml}$ ketamine and $2.6 \mathrm{mg} / \mathrm{ml}$ xylazine) and secured on the microscope stage in a supine position using rubber bands holding the forelimbs and the incisors. Each animal was intubated and its respiration was mechanically controlled by a ventilator (Harvard Apparatus; model 683). Hair on the ventral neck was removed using depilatory cream. Using aseptic procedures, the left sternomastoid muscle was exposed by a midline incision. The postsynaptic acetylcholine receptors (AChRs) were then labeled with rhodamine-conjugated bungarotoxin (rho-BTX) (Invitrogen; $5 \mathrm{~min}$ of $2 \mu \mathrm{g} / \mathrm{ml}$ in sterile lactated Ringer's, followed by rinsing with the same Ringer's). Given the large safety factor of the neuromuscular junction in adults $(>70 \%$ of AChRs must be blocked to disrupt transmission), this dosage does not block postsynaptic activity (Lingle and Steinbach, 1988). The sternomastoid muscle, immersed in sterile lactated Ringer's, was slightly elevated by a stainless-steel ring placed underneath the muscle and attached to a small manipulator. A similar ring was placed on the ventral surface of the muscle surrounding the central band of neuromuscular junctions to both stabilize and slightly flatten the muscle. Images were captured with a Nikon $40 \times(0.8 \mathrm{NA})$ immersion objective attached to a Leica DMR epifluorescence microscope using filter sets described previously (Zuo et al., 2004). The $100 \mathrm{~W}$ mercury fluorescence excitation was attenuated to one-eighth of its intensity by neutral density filters and exposure time limited to $1 \mathrm{~s}$. Images were collected using a cooled CCD camera (CoolSnap HQ; Photometrics), $2 \times 2$ binning, and IPLab3.5 software on a Macintosh computer. Images were acquired during brief periods in which the ventilator was turned off and the animal was not making respiratory movements. If all features could not be seen in a single focal plane, images were collected in a few planes and then manually montaged together (as can be discerned in some of the illustrations below). After all images were collected, the wound in the skin was sutured and the animal was allowed to fully recover in its home cage under a heat lamp. The same junctions were reimaged at subsequent time point(s) by repeating this procedure. Images were analyzed using the IPLab software and Adobe Photoshop. Adjustments were made to image brightness and contrast.

Laser ablation. Preceding the capture of the first image in each animal (and only at this time), a solution of the fluorescent dye 4-Di-2-Asp (0.1 $\mu \mathrm{g} / \mathrm{ml}$ in lactated Ringer's) was applied for $1 \mathrm{~min}$ after the application of the rho-BTX (Magrassi et al., 1987; Rich and Lichtman, 1989a). This dye provided a background labeling of the muscle fibers and facilitated identification of a length of the fiber whose ablation was desired. Ablation of individual fibers was accomplished using a Zeiss epifluorescence microscope with a commercial laser ablation attachment (MicroPoint; Pho- tonic Instruments). A $2.4 \mathrm{~mW}$ nitrogen laser (model VSL-337; Laser Science) was used to pump a dye cell containing coumarin 480 . The resulting laser light was focused through a $63 \times, 0.9 \mathrm{NA}$ water objective, and its intensity adjusted by a neutral density attenuator. The ablation was monitored through a SIT camera attached to the microscope and a TV monitor. Each candidate fiber on the surface of the muscle, selected because the labeled AChR in its NMJ presented an en face view, was irradiated three to four times on each side of the junction, but the endplate itself was not targeted. A plasma bubble, indicative of the interaction of the laser microbeam with the target (van Mier and Lichtman, 1994), was always visible at the site of irradiation at the time of the ablation. This method essentially duplicates that first applied by van Mier and Lichtman (1994).

Other live staining. Propidium iodide $(0.1 \mathrm{~mm})$ (selectively labels dead cell nuclei) was applied to the living muscle for $3 \mathrm{~min} 1 \mathrm{~h}$ after laser irradiation (Jones and Senft, 1985; van Mier and Lichtman, 1994).

Wide-field and confocal microscopy of fixed preparations. At the time of the terminal experiment after the final vital images were collected, animals were killed with pentobarbital $(0.05-0.1 \mathrm{ml}$ of $5 \mathrm{~g} / \mathrm{ml})$. The muscle was then dissected and fixed in $4 \%$ paraformaldehyde in PBS, $\mathrm{pH} 7.4$, for $30 \mathrm{~min}$ and rinsed with PBS for three times, 10 min each. A thin layer of surface fibers was then dissected from the whole muscle and mounted in fluorescence mounting medium. The same endplates imaged in vivo were located in vitro, based upon the label for AChRs.

In some nonvital experiments, the muscle was incubated with $4^{\prime}, 6$ diamidino-2-phenylindole (DAPI) $\left(10^{-4} \mathrm{mg} / \mathrm{ml}\right)$. In other experiments, the muscle was incubated with Alexa 647-conjugated fasciculin II, a snake toxin that binds to acetylcholinesterase (AChE) (Peng et al., 1999). The toxin (Sigma-Aldrich; F4293) was conjugated to the dye using a microscale protein labeling kit (Invitrogen; A-30009). For antibody labeling of AChR $\beta 1$-subunit, we used a monoclonal rat anti-Torpedo antibody (at 1:2000 dilution; Sigma-Aldrich; N8283) directed against an intracellular epitope. This was then followed by applying Alexa 594 goat anti-rat secondary (1:250; Invitrogen; A-11007). In experiments in which a second color of bungarotoxin was applied nonvitally to muscles previously vitally labeled with rhodamine bungarotoxin and the 647 channel was used by AChE labeling, an Alexa 594 bungarotoxin was used and the colors separated by confocal microscopy.

These preparations were imaged with a Leica DMR microscope with a $40 \times(1.0 \mathrm{NA})$ oil lens. Confocal images were acquired using a Leica confocal microscope (Leica; AOBS SP2) and a 63×, 1.4 NA oil objective. Images were analyzed using Leica confocal software, NIH ImageJ, MetaMorph (Molecular Devices), and Imaris (Bitplane).

Physiology. The sternomastoid muscle was rapidly dissected from a killed animal and pinned to a Sylgard-coated dish. The muscle was superfused at all times with oxygenated Ringer's solution containing $2 \mathrm{~mm}$ $\mathrm{Ca}$ (Liley, 1956). Microelectrodes filled with $3 \mathrm{M} \mathrm{KCl} \mathrm{(10-40} \mathrm{M} \Omega$ in resistance) were inserted into the endplate regions of muscle fibers using a micromanipulator, and the visual guidance was provided by waterimmersion objectives and a Zeiss Axiotech epifluorescence microscope. Endplates were identified by labeling with rho-BTX. The membrane potential was monitored using a high-impedance amplifier (Axoclamp 2B; Molecular Devices) and an oscilloscope (Tektronix).

\section{Results}

\section{Laser light can be used to photoablate the muscle fiber} beneath individual NMJs in living mice

The postsynaptic muscle fiber at individual NMJs in the sternomastoid muscle of mice was ablated with a laser microbeam. The ablation was performed while vitally imaging the synaptic components using wide-field fluorescence microscopy, a procedure originally developed by van Mier and Lichtman (1994). A nonblocking concentration of rho-BTX was used to label a fraction of the acetylcholine receptors at the NMJs in the red fluorescence channel; vital, transgenic expression of CFP (Feng et al., 2000) and of GFP (Kang et al., 2003) labeled the motor axon terminal in a blue channel and the Schwann cells in a green channel, respectively. A vital, fluorescent mitochondrial dye (4-Di-2-Asp) 
(Magrassi et al., 1987; Rich and Lichtman, 1989a) was applied to provide a background labeling that revealed the outline of the muscle fibers (also in the red channel). Laser light pulses at 480 $\mathrm{nm}$ were then applied to sites located on each side of the NMJ. These sites (six to eight of them per fiber) were positioned $100-$ $150 \mu \mathrm{m}$ away from the junction itself and spaced at $100 \mu \mathrm{m}$ intervals along both sides of the NMJ. We were successful in the ablation of 211 muscle fibers on each side of the NMJ in the sternomastoid muscle of 68 mice.

Several assessments show that the muscle fiber underneath the nerve terminal was ablated in these experiments: (1) The 4-Di-2Asp fluorescence, representing the background of the components within the muscle fiber, was lost almost immediately from the site of laser application and from the fiber underneath the junction within $10 \mathrm{~min}$ (data not shown). A "contraction clot" (van Mier and Lichtman, 1994) in which the dye-labeled components moved into clumps on each side of the site of ablation was seen (data not shown). (2) Propidium iodide (PI), a membraneimpermeant nuclear dye (Jones and Senft, 1985; van Mier and Lichtman, 1994), was applied to a sample of 11 ablated fibers $1 \mathrm{~h}$ after the attempted ablation in three muscles to determine whether the sole plate nuclei (the muscle nuclei underneath the nerve terminal) could be labeled (Rich and Lichtman, 1989a). The animal was then killed, the muscle was fixed, and DAPI was applied to label all nuclei present. We compared the number of nuclei with each label that were located within $10 \mu \mathrm{m}$ of the endplate and positioned beneath the Schwann cells and AChR. Every obvious sole plate nucleus labeled with DAPI was also labeled with PI (data not shown). Moreover, the number of nuclei with PI label ( $4.3 \pm 1.0 ; n=11 \mathrm{NMJs})$ was similar to the number of DAPI-labeled sole plate nuclei present in undamaged control endplates ( $4.2 \pm 1.2 ; n=14 \mathrm{NMJs})$. The nuclei of Schwann cells present at these sites, identified by their GFP expression, were labeled by DAPI but, as expected since they were not intentionally damaged, were unlabeled by PI. An occasional DAPI-positive nucleus unlabeled by PI that was not a Schwann cell was encountered; these are probably the nuclei of fibroblasts in the vicinity of the junction (Gatchalian et al., 1989; Ribchester, 2009). We conclude that muscle fiber nuclei at the endplate of these damaged fibers were made accessible to PI by the damage inflicted by the laser. (3) Microelectrodes were used in attempts to penetrate the laser-targeted fibers $1 \mathrm{~d}$ after laser ablation. No membrane potential could be detected when the area just beneath the junction was impaled (nine ablated fibers in three animals), but such potentials were easily detected at control junctions. (4) We performed the same type of ablations on two muscle fibers in a single mouse lacking the transgenes described above but whose macrophages and other immune cells are transgenically labeled with YFP (S100-YFP line YH; Zuo et al., 2004). Examination of the muscle $1 \mathrm{~d}$ later revealed that YFP+ cells had accumulated underneath the labeled AChR, within the volume of the damaged fiber (Fig. $1 \mathrm{~A}$ ), and in the immediate area surrounding the fiber. (5) In eight of eight cases of attempted ablation in which we stained for nuclei with the fluorescent dye DAPI 6-9 d later, we found that the site of the ablated fiber, clearly identifiable by its distinct pattern of AChR (as well as by nerve terminal branches and Schwann cells, see below), was occupied by a new fiber that had chains of centrally located nuclei (data not shown). Since such central nuclei define muscle fibers regenerating following injury (Duchen et al., 1974), we conclude the laser had caused the original fiber to degenerate and a new fiber/fiber segment to form in its place.

\section{AChR labeling declines rapidly at the synaptic site on ablated fibers}

Because of its avid binding to AChR, BTX can be used to monitor the turnover of these receptors at living neuromuscular junctions (Akaaboune et al., 1999). A fluorescently tagged version of the toxin is applied at a concentration that is nonblocking and the decline in fluorescence over time was observed. At normal, adult neuromuscular junctions in rodents, the decline in this fluorescent signal indicates a turnover proceeding with a half-life of $\sim 2$ weeks (Akaaboune et al., 1999). Previous studies of the consequences of fiber ablation have reported that there is a rapid loss of AChR from the synaptic site (Jirmanová, 1975; Marshall et al., 1977; Burden et al., 1979; Couteaux et al., 1988; Rich and Lichtman, 1989a; Dunaevsky and Connor, 1998), presumably as the receptors in the damaged muscle membrane are destroyed or phagocytosed. To confirm these predictions in our preparation, we labeled receptors in the sternomastoid muscle with nonblocking concentrations of rho-BTX and then followed the fluorescence signal over time at the same junctions, some of which had undergone ablation of their muscle fibers. Junctions were imaged immediately following application of BTX and then reimaged 2, $6,12,20$, and $30 \mathrm{~d}$ later (Fig. $1 B, C$ ). In none of these particular experiments was BTX applied subsequent to the first imaging session. As expected (Akaaboune et al., 1999), the labeling intensity on un-ablated fibers declined gradually (data not shown). Approximately $21 \%$ of the intensity present in the initial image was gone $2 \mathrm{~d}$ later. There was no obvious change in the pattern of the AChR in the muscle membrane on these un-ablated fibers, consistent with the known stability of the neuromuscular synaptic contact in young adult mice (Lichtman et al., 1987). As also expected from previous ablation studies (Rich and Lichtman, 1989a; van Mier and Lichtman, 1994), there was a far more abrupt decline in the labeling intensity of the receptors on junctions following laser damage to the fibers (Fig. $1 B$ ): $\sim 60 \%$ of the label present at the initial time point was gone $2 \mathrm{~d}$ later. The junctions on the ablated fibers continued to become dimmer, so that, by $6-12 \mathrm{~d}$, no labeling was obvious in the unenhanced images acquired by the CCD camera (Fig. $1 B$ ). At the same time, faint labeling was still visible at the junctions on un-ablated fibers (data not shown). Thus, AChRs on ablated fibers disappear much more quickly than they turn over at intact neuromuscular junctions.

\section{Some AChR present at the time of ablation and AChE persist for some time and define the original site of the synaptic contact}

Even though the unenhanced images showed the AChRs were disappearing from the ablated fibers (Fig. 1B), enhancement of these images by adjustment of contrast and brightness clearly showed a persistence of some fluorescent label (Fig. 1C; see also Fig. 4); this label persisted for periods of as long as 11 weeks (data not shown). This persistent labeling initially has the form/outline of the AChR "pretzel" present before ablation, but is shrunken and distorted in shape, most prominently in the cross-fiber axis [Fig. 1C; see also Rich and Lichtman (1989a), their Fig. 6]. The likely explanation for this shrinkage/distortion is the collapse/ folding of the basal lamina that previously surrounded the muscle fiber and extended through the synaptic cleft (Marshall et al., 1977). Major features in the pattern seen before ablation persist, as do minor features when they are not obscured by the distortions. Such persistence of the original label was also obtained when sternomastoid fibers were ablated by mechanical damage 

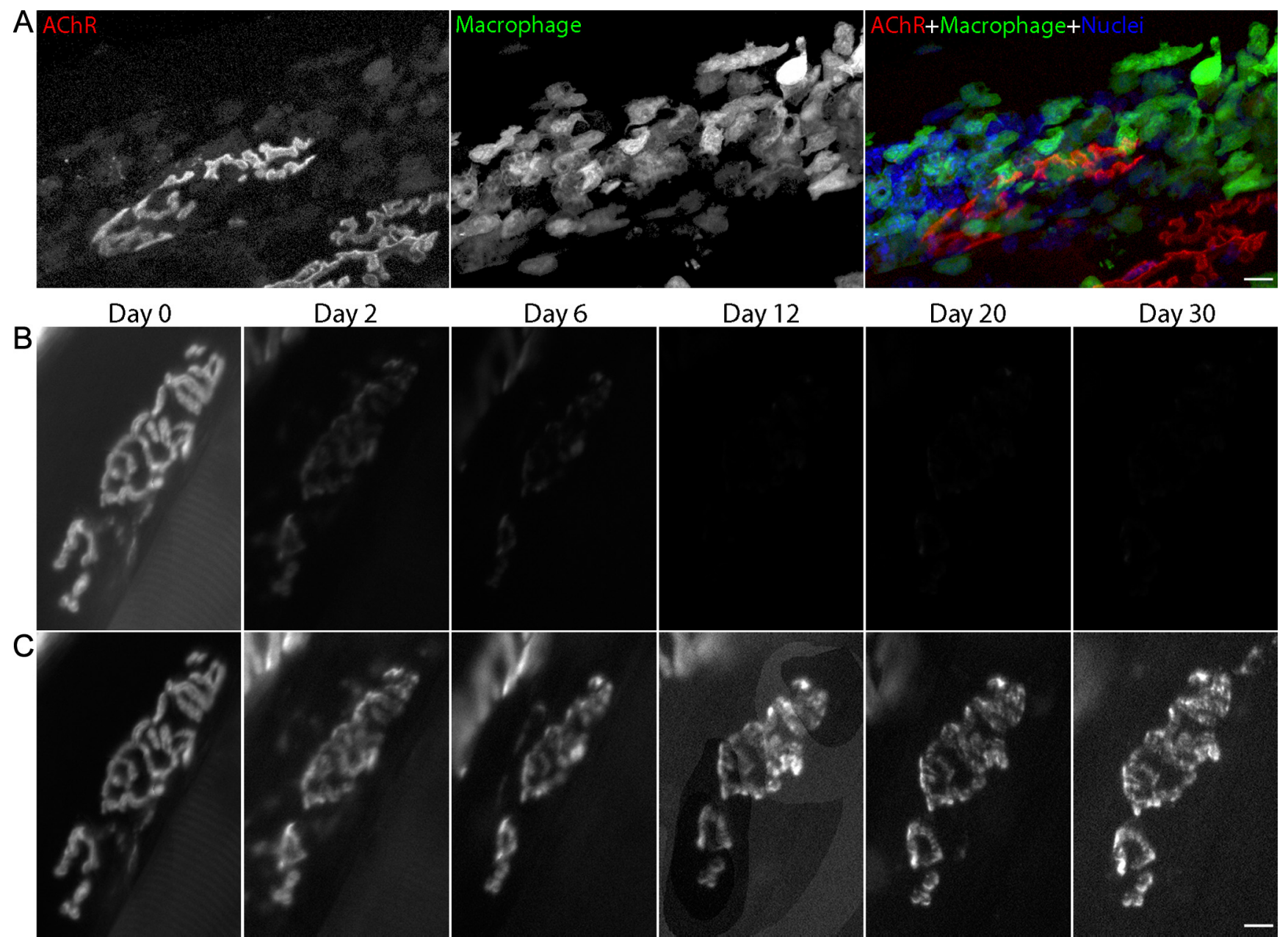

Day 12
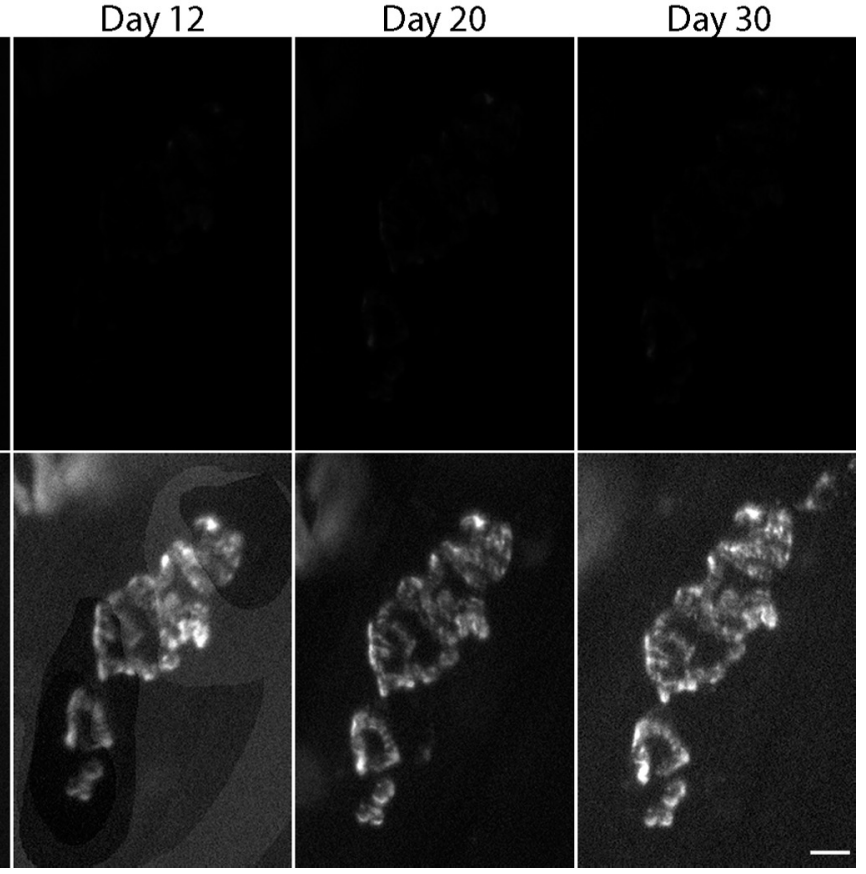

Figure 1. Muscle fiber ablation leads to a rapid, dramaticloss of AChRs, buta low level of AChRs persists for some time. $A$, Transgenicmouse with YFP expression in macrophage and other immune cells shows the accumulation of YFP + cells within and around the ablated muscle fiber $1 \mathrm{~d}$ after ablation. The ablated fiber is identified by the fingerprint of its AChR labeled by rho-BTX (leftmost panel) and imaged at the time of ablation (data not shown). B, AChR labeled with a single application of rho-BTX before ablation and then imaged successively, immediately after ablation and then at intervals extending to $30 \mathrm{~d} \mathrm{later}$. All the images were taken with identical illumination and at the same exposure and camera gain as the first panel; all these images are unenhanced in software. The intensity of the label quickly becomes so low that no fluorescence is detected by day 12. C, Enhancement of the same images as in $\boldsymbol{B}$ shows that a faint labeling persists at the synaptic site; a greater enhancement in each successive image is performed to make the intensity appear like that in the first panel. The labeling initially shrinks and becomes spotty, but the same pattern persists. Scale bars: $A-C, 10 \mu \mathrm{m}$.

with iridectomy scissors on each side of the endplate (two fibers examined at $6 \mathrm{~d}$ after injury; data not shown).

AChE attached to the basal lamina at the synaptic site has been previously used as a marker for the original synaptic site on damaged muscle fibers (McMahan et al., 1978). We used fluorophore-conjugated fasciculin-2 (Peng et al., 1999), a small molecule component of snake venom that binds tightly and selectively to the catalytic subunit of AChE to label the synaptic basal lamina. To avoid the possibility that the blockade of AChE by this toxin would itself alter the synapse, we did not use this reagent as a vital stain but rather used it at the time of the terminal experiment. We found that the low-intensity AChR label obtained with BTX colocalized with this AChE during the first $3 \mathrm{~d}$ after fiber ablation (see Fig. 3). The colocalization was apparent at all levels in the confocal $z$-slices through these ablated fibers. The colabeling shows that these persistent receptors somehow remain closely associated with the old basal lamina, despite, as reported by others (Duchen et al., 1974; Jirmanová, 1975; Couteaux et al., 1988), the loss of the sarcolemma. To further evaluate the possibility that the persistent fluorescence from the BTX might not be associated with $\mathrm{AChR}$, we compared the labeling at $3 \mathrm{~d}$ after laser ablation with the labeling obtained with an antibody directed against an intracellular epitope of the $\beta$-subunit of the receptor. The two labels coincide on the ablated fibers $(n=6)$, just as they do on control, un-ablated fibers (data not shown). These findings are in agreement with previous reports of a persistence of BTX label in other cases of muscle fiber ablation (Burden et al., 1979; Slater and Allen, 1985; Rich and Lichtman, 1989a). In addition, a recent study in vitro suggests that receptors that form a pretzel-like aggregate in the membranes of muscle fibers grown in contact with an artificial basal lamina persist in association with the extracellular matrix after destruction of the muscle membrane (Kummer et al., 2004). Thus, the weak labeling of receptors with bungarotoxin applied at the time of ablation defines the previous synaptic site on the basal lamina persisting following degeneration of the muscle fiber.

\section{Despite degeneration of the muscle fiber, the nerve terminal} and Schwann cells initially retain their original configurations and remain associated with the former synaptic basal lamina

In addition to examining the distribution of receptors, we also sought to determine the disposition of the nerve terminal and the 

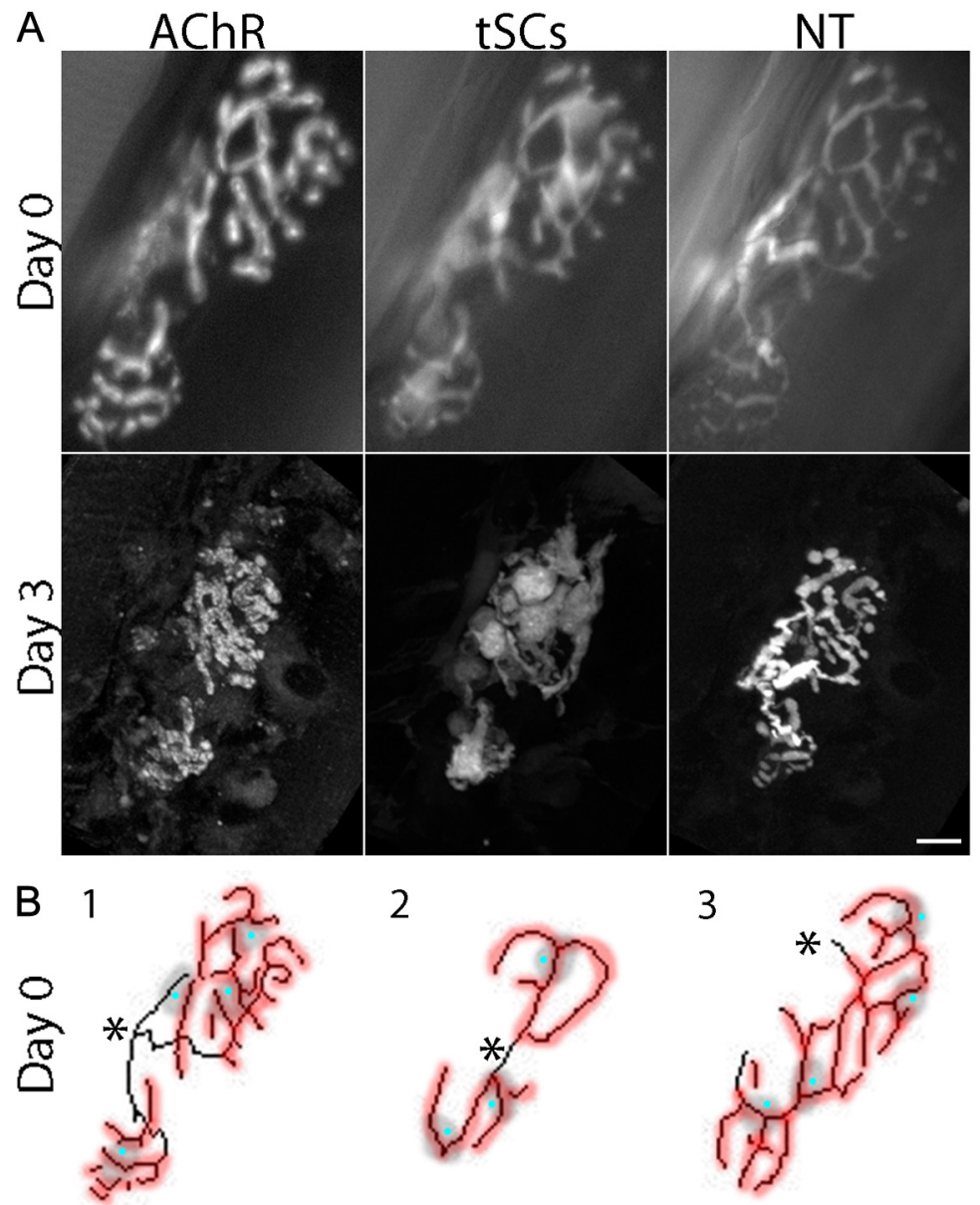

2

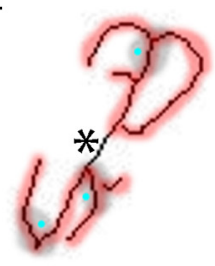

3
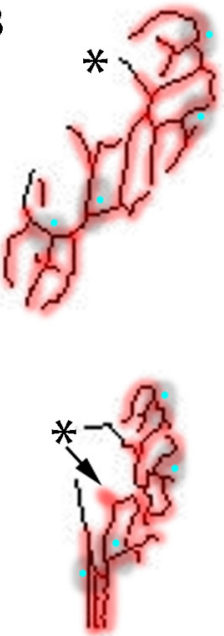

Figure 2. Vital and nonvital images collected $3 \mathrm{~d}$ after fiber ablation show little change in the nerve terminal branches and $\mathrm{SCS}$ associated with persistent AChRs. A, Vital, wide-field images of AChR (rho-BTX), tSCs (transgenic GFP), and motor nerve terminals (transgenic CFP) collected immediately after ablation (day 0 ) and nonvital, confocal images collected $3 \mathrm{~d}$ later. The confocal images were collected as a stack and the stack rotated to present the original en face image and a maximum projection made. The nerve terminals and SCs remain in association with the AChR that persist. Given the resolution of the initial vital images, the resemblance of the patterns is remarkable. $\boldsymbol{B}$, Drawings of three synaptic sites both before (day 0 ) and $3 \mathrm{~d}$ after ablation of the muscle fiber. Red is used to show the AChR; the nerve terminals are drawn in black lines and the cell bodies of the $\mathrm{SC} C \mathrm{~S}$ as gray ovals with central dots. Drawing 1 is the same junction as in $A$. Drawing 2 shows one junction with a small sprout at day 3 (arrowhead). Drawing 3 shows one junction with a small area of AChR at which no nerve terminal was detected (small arrow). The sites, although distorted and shrunken, show no evidence of any dramatic change in the nerve terminals or the associated $\mathrm{SC}$ S. Two other junctions (data not shown) gave similar results. The asterisk in each drawing indicates the site of entry of the preterminal axon. Scale bar: $10 \mu \mathrm{m}$ (all panels).

associated Schwann cells within the first few days after fiber ablation. We did this both in junctions imaged vitally and in junctions in which the muscles were examined in vitro and stained for cholinesterase at set times postablation. Despite difficulties presented by shrinkage and changes in the orientation of the fibers, we believe that the nerve terminals and Schwann cells are very little affected during the first $3 \mathrm{~d}$ following fiber ablation and that the nerve terminals most commonly remain in contact with the old synaptic basal lamina. This conclusion is based on the following sets of observations.
First, we obtained a number of vital images 2-3 d following ablation in which the sites remained en face or at least a large portion of the original site did so. In $71 \%$ of the junctions (10 of 14) whose complete configuration was identifiable $2 \mathrm{~d}$ after fiber damage, the whole apparatus was just a smaller version of that present before ablation (data not shown). In the remaining 4 of these 14 junctions, the nerve terminal had lost only one to three small branches that occupied on average only $3 \pm 1 \%$ (mean \pm SEM) of the receptor area (each branch of the original axon terminal was measured and compared with the image $2 \mathrm{~d}$ later; data not shown). No terminal sprouts were visible at this time (van Mier and Lichtman, 1994). The number of Schwann cell bodies and their approximate location were unchanged, and none of the terminal Schwann cells (tSCs) had extended processes that are known to grow after the same period of denervation (Son and Thompson, 1995).

To obtain a set of fibers on which we could more thoroughly examine the nerve terminal and the Schwann cells for alterations that might accompany the disappearance of the postsynaptic muscle fiber, we ablated five fibers in two young adult mice, vitally imaged the junctions $3 \mathrm{~d}$ later, but then killed the animals, fixed their muscles, found these same fibers in a layer of muscle dissected from the surface of the muscle, and examined these fibers in the confocal microscope. Image stacks were prepared and each junction visualized in 3D. This enabled the rotation of the stack to obtain an en face view of the junction similar to the one made immediately following laser ablation. The results were quite striking (Fig. $2 A$ ): for all five junctions, the number, order, and pattern of the branches were quite similar. With two very small exceptions (representing $<3 \%$ of the old area of contact outlined by the persistent BTX labeling, some of which are indicated in Fig. $2 B$ ), the nerve terminals remained in apposition to material that, as argued above, delineates the old synaptic site.

In still another set of mice, we ablated muscle fibers and examined the same junctions on these ablated muscle fibers $3 \mathrm{~d}$ later, in this case labeling the AChE with fasciculin-2 (Fig. 3). We saw in each of three junctions that the nerve remained closely associated with the entire extent of the cholinesterase label and with the persistent AChR label. However, given the resolution of the light microscopy here, we cannot exclude the possibility that there are areas where there are gaps between the nerve terminal and the AChE labeling that are not apparent in our images. Here as before, Schwann cells had not sprouted processes away from the junction. 


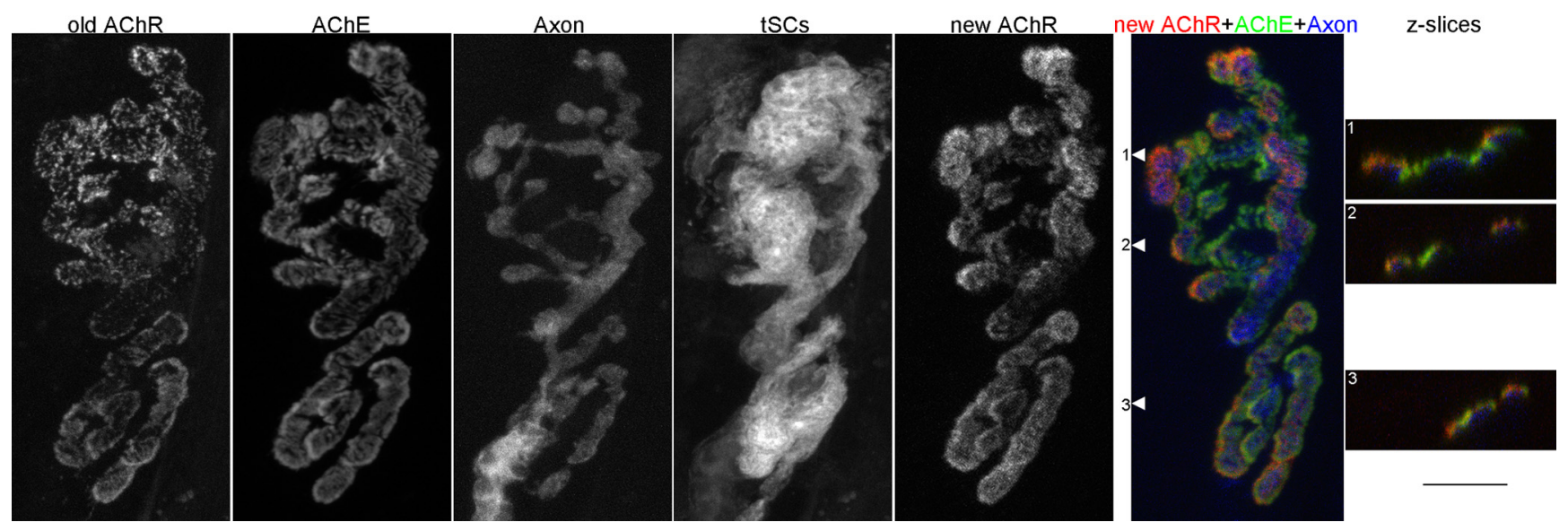

Figure 3. The nerve terminal remains in contact with the synaptic basal lamina $3 \mathrm{~d}$ following fiber ablation with no apparent alterations. Confocal views of a NMJ whose muscle fiber was ablated 3 dearlier. At the time of ablation, the AChRs were labeled with rho-BTX ("old AChR"). At $3 \mathrm{~d}$, after dissection and fixation, the sternomastoid was labeled with Alexa 647-conjugated fasciculin 2, a toxin with high affinity for AChE. The grayscale images to the left show the maximum projections of the labeling for old receptor, AChE, the transgenic CFP in the axons, and the transgenic GFP in the SCs, respectively. The maximum projection in color shows the old AChR (red), the AChE (green), and the axon (blue). The panels to the right show z-slices of this last maximum projection. The nerve, the AChE, and the AChR all colocalize, showing that the nerve terminal continues to occupy the old synaptic basal lamina. For this particular fiber, there had been regeneration of the postsynaptic fiber and new AChRs and DAPI-stained central nuclei were present as well (data not shown). The junction shown here is typical of that observed in three total cases. Note absence of extensive SC extensions beyond the AChE. Scale bar: $10 \mu \mathrm{m}$ (all panels).

These experiments show that, except for shrinkage and some minor losses, the nerve terminal and Schwann cells retain their original positions and branching and are apposed to persistent receptors at mouse NMJs during the first $3 \mathrm{~d}$ following the ablation of their postsynaptic targets. Thus, the nerve terminal is not remodeled as a consequence of the loss of the postsynaptic target. These results also argue that the synaptic basal lamina remains intact and is not itself damaged during the events of fiber ablation. Stability of the nerve terminal following ablation of the target muscle has been reported for the frog NMJ (Dunaevsky and Connor, 1998). While a previous study in mouse reached a different conclusion (Rich and Lichtman, 1989a), we believe the discrepancy here is likely accounted for by use of a vital label that gives a less complete view of the nerve terminal arbor than does the cytoplasmic CFP used here. In frogs, the targetless nerve terminals remain grossly unaltered for weeks (Yao, 1988; Dunaevsky and Connor, 1998). We do not know whether this is the case in the mice, as this would require inhibition of muscle fiber regeneration as was done in the frog.

\section{New AChRs appear upon regenerating muscle fibers}

Muscle fibers that are damaged and undergo segmental necrosis (Papadimitriou et al., 1990) regenerate from the activation of satellite cells and their fusion into the fiber (Le Grand and Rudnicki, 2007). Commonly, these fibers regenerate within the basal lamina tube that persists following degeneration of the preexisting fiber (Carlson, 1973). Previous studies show that fibers began to regenerate in the mouse 2-3 d after fiber damage (Harris and Johnson, 1978). In our material, the outline of a thin myotube was sometimes visible at the injured site on day 4 , although sarcomeric striations, commonly visible with labeling with 4-Di-2Asp, were not detectable at this time (data not shown). When preparations were stained with DAPI, myotubes containing chains of "central nuclei" were seen to be associated with the NMJs identified as those present on the ablated fibers (see above).

Previous study has shown that, as muscle fibers regenerate, they add AChRs to the synaptic sites that form upon them (Rich and Lichtman, 1989a; Lyons and Slater, 1991). If the nerve is absent, AChRs still accumulate in the muscle fiber membrane as dictated by agrin localized to the synaptic basal lamina (McMahan and Slater, 1984). To examine the AChRs on the regenerating muscle fibers in our experiments, we applied, subsequent to the first images taken at the time of fiber ablation, a second fluorescent color of BTX (Alexa 647-BTX, at the same concentration and for the same length of time as the first color). Vital imaging sessions (2, 4, 6, and $12 \mathrm{~d}$ after ablation) showed initially (at day 2), only a very faint labeling with this second-color BTX; this faint labeling aligned uniformly with the persistent, first-color bungarotoxin (data not shown). Since the fiber had not regenerated at this time, we interpret this faint labeling with the second-color BTX as labeling of a small number of persistent receptors that were present at the time of the labeling with the first-color BTX but that had been unlabeled by the first application. However beginning at 3-4 d after ablation and the reapplication of the second-color BTX, some intensely labeled foci were found in close proximity to a few of the sites with persistent receptors (Fig. $4 B 2$ ). The intensity of the label in these foci, in contrast to the faint labeling of persistent receptors, shows these are sites where new receptors were deposited postablation. When the same junctions were reimaged $2 \mathrm{~d}$ later (at $6 \mathrm{~d}$ ) and the second-color BTX reapplied, the intensity of the label had increased and additional new areas of labeling had been added, in many cases linking the previous foci and making the labeling more uniform (Fig. 4B3). Following an additional $6 \mathrm{~d}$ (12 d following ablation), the application of the second-color BTX yielded an even more intense label, but the number of new areas added had dropped significantly (Fig. 4 B4 ). These observations suggest a continuing deposition of additional AChRs into the membrane of the regenerating muscle fibers. The number of additional new sites formed seems to reach a peak around $6 \mathrm{~d}$ and then decline (see below).

\section{Synapses established on regenerating fibers deviate} significantly from those expected from the old synaptic basal lamina

The synapses that form on the regenerating fibers do not recapitulate the previous synapse as would be expected if they formed solely under the influence of the old synaptic basal lamina. 

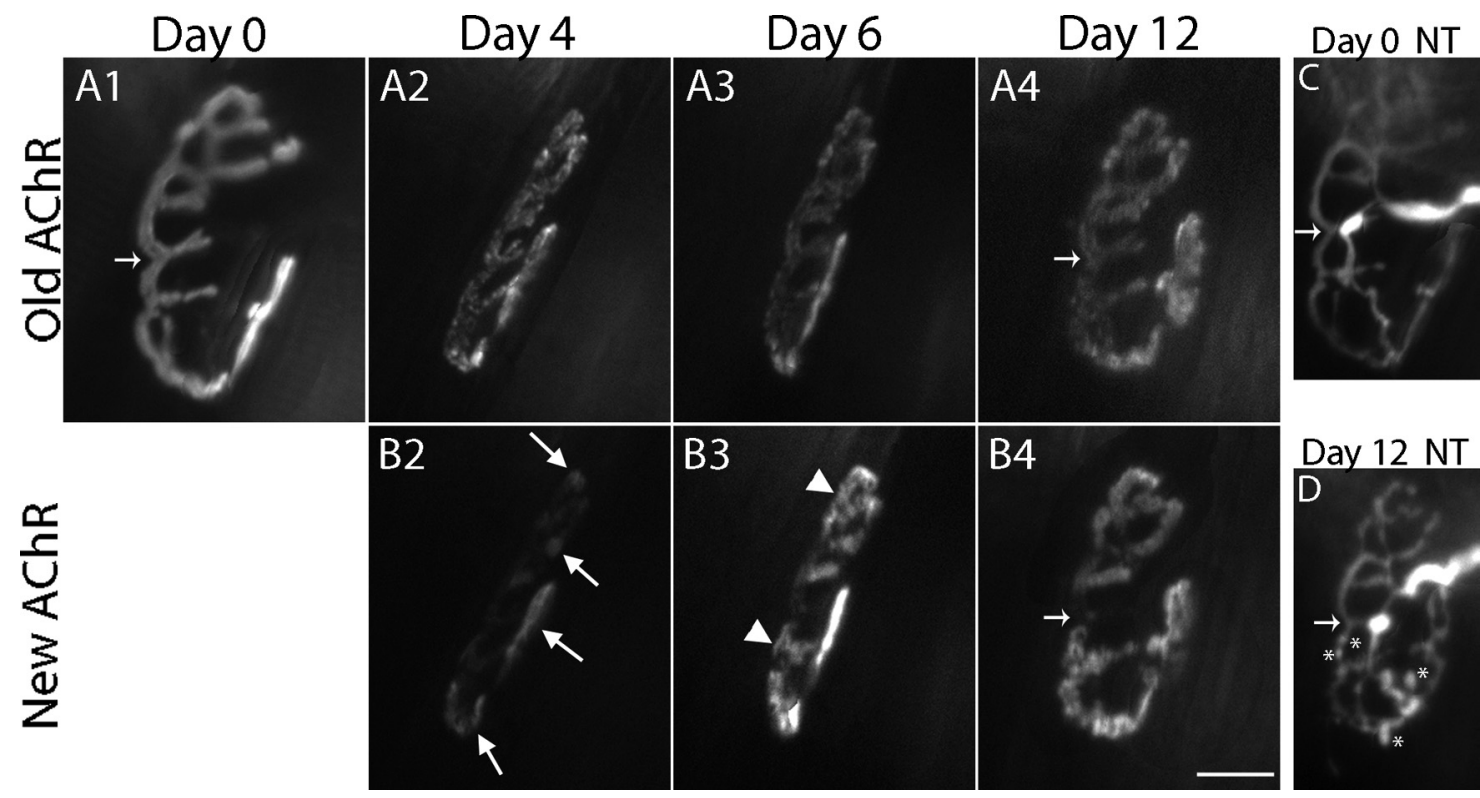

Figure 4. New receptors appear gradually at the synaptic site on the regenerating muscle fiber. $A$, Rho-BTX was applied before ablation of the muscle fiber underneath this junction, and this same label (without application of any additional BTX) followed in three additional imaging sessions over the subsequent $12 \mathrm{~d}$. The images on days $4-12$ (A2-A4) were enhanced in contrast and brightness to match the intensities seen in $\boldsymbol{A}$. Although distorted by shrinkage of the fiber, the pattern of receptors is remarkably similar to that at the time of the initial image. $\boldsymbol{B}, A$ second color of BTX (Alexa 647) was applied on day 4 and again on each of the subsequent imaging sessions on days 6 and 12 . The contrast and brightness of the images in $B 2-B 4$ are the same, but are augmented over that in $\boldsymbol{A}$ 1. Labeling with the second-color BTX appears in foci (B2, arrows); these spots intensify and expand ( $\boldsymbol{B} 3$, arrowheads), creating a pattern at day 12 (Bי) that resembles remarkably the pattern in $\boldsymbol{A} \mathbf{1}$ and $\boldsymbol{A 4}$, with some exceptions (an obvious absence of new receptors is indicated by the small arrow in $\boldsymbol{B} \mathbf{4}$ ). $\boldsymbol{C}$, Nerve terminal (marked by transgenic (FP) at time of fiber ablation. $\boldsymbol{D}$, Nerve terminal at day 12 . Comparison of $\boldsymbol{A} \mathbf{1}, \boldsymbol{A} \boldsymbol{4}, \boldsymbol{B} \boldsymbol{4}, \boldsymbol{C}$, and $\boldsymbol{D}$ shows that the loss of receptor areas in $\boldsymbol{B} \mathbf{4}$ (small arrow) is not due to loss of a nerve terminal process in this area (small arrow marks the same position in $A \mathbf{1}, A 4, B 4, C, D)$. Small processes emerge from the nerve terminal $12 \mathrm{~d}$ after ablation ( $D$, asterisks) and the terminal appears more varicose than seen initially. When examined next at day 28 , the $A C h R$ in $\boldsymbol{B} 4$ were highly fragmented (data not shown). Scale bar, $20 \mu \mathrm{m}$.

Rather, the new synapses differ significantly in their morphology from the old ones in several ways. To examine these differences in more detail than is possible in the vital imaging experiments described above, we imaged junctions labeled in vivo with a first color of BTX, ablated the fibers underneath these junctions, and then allowed 3 or $6 \mathrm{~d}$ to elapse before the animal was killed, the muscle fixed, and a second color of BTX applied to label any new receptors deposited at the synapse. In some cases, fasiculin-2 was applied to label the cholinesterase that would be expected to define the original synaptic site for a period following regeneration. The junctions on the ablated muscle fibers were relocated in the confocal microscope and image stacks collected. In this way, we could rotate the images if the fiber had changed from an en face presentation of the junction, and we could examine the relative locations of the various labels. We consistently find the following types of differences.

First, we find there are branches of the nerve terminal that were previously synaptic that do not come into contact with the regenerating fiber, likely because of folding of the old basal lamina. In confocal slices, these portions of the nerve terminal never obtain new AChRs and are found in confocal slices to be located above the surface of the regenerated fiber. A particularly clear example of this is shown in the drawing of Figure $5 B 2$, where a long terminal branch connected to synaptic contacts is itself no longer synaptic. That such branches were previously synaptic is shown by the old, labeled AChRs that are associated with them. An example in the vital images that is likely explained by this kind of change is seen in Figure 4.

Second, the nerve terminal begins to grow short extensions that reach the surface of the regenerating fiber and form synaptic contacts outside the areas of the original contact. These types of changes are obvious from observations of the deposition of new
AChRs at sites adjacent to, but clearly separate from the location of old receptors (Fig. 5). Furthermore, in the cases in which AChE label was applied to the muscle after its dissection from the animal, the nerve had extended beyond the cholinesterase and were contacting sites where new AChR were located (Fig. 6). Schwann cell extensions were associated with these nerve terminal growths, being either longer or coextensive.

Third, from gross inspection within the area of the old synaptic site, it appeared that the new receptors were commonly being deposited in the same locations as the old receptors and the cholinesterase (Fig. 6). However, confocal sections through these junctions revealed that in many cases the new receptors were displaced beneath the cholinesterase toward the regenerating fiber. In these cases, it appears from the CFP label that the nerve has penetrated through the basal lamina, grown toward the regenerating muscle fiber, and made synaptic contact before the fiber has grown into contact with the basal lamina. Indeed, we saw that holes had appeared in the cholinesterase label at $3 \mathrm{~d}$ following ablation and at $6 \mathrm{~d}$, after the fiber had begun to regenerate, many of these holes were sites where small extensions of the nerve [and SCs (data not shown)] were present extending toward the fiber center (Fig. 6). These holes appear to form even in cases in which the muscle fibers were ablated and the muscle denervated by resection of the muscle nerve (three ablated fibers; data not shown). Thus, the holes are likely formed by the phagocytes that invade to remove components of the ablated muscle or possibly by the SCs that remain at the site rather than the nerve itself. Interestingly, the holes do not appear to develop on fibers that are denervated but not ablated. Many of the sites of new receptor deposition were indistinguishable in their location in the confo$\mathrm{cal} z$-stacks from the AChE/old receptor. These could be possibly generated from signals originating from the old synaptic basal 
lamina. This is supported by the observation that in regenerating muscle fibers that have been denervated, the new receptor and AChE colocalize (data not shown; three junctions in one muscle at $6 \mathrm{~d}$ following fiber ablation and denervation), suggesting that the remodeling reported above requires the presence of the nerve. This finding supports previous observations (Rich and Lichtman, 1989a; Marques et al., 2005). The absence of remodeling of new receptors in the absence of the nerve also argues that the changes we report above are not due to damage of the basal lamina during ablation of the muscle fiber. Some of the cases of colocalized new receptors and AChE in the innervated, ablated fibers might be instances in which the distance separating new and old falls beneath the resolution of the light microscope.

Last, the synaptic sites began to undergo a period of remodeling, as described in the following section.

\section{NMJs on regenerated fibers continue to} remodel following their formation As reported previously (Rich and Lichtman, 1989a), the synapses on regenerating muscle fibers undergo remodeling, unlike the NMJs on adjacent fibers that were not ablated. To document these changes in our preparations, we repeatedly imaged a set of six NMJs in two mice out to $80 \mathrm{~d}$ after ablation in one case and $133 \mathrm{~d}$ in the other. One of these is illustrated in Figure 7. The junctions undergo the early changes described above. At day 6 , the AChR labeled at the time of ablation are greatly diminished in intensity (Fig. 7, compare $A 1, B 1$ ). When examined at day 12, new AChR are present, but the AChR appear as fragments, rather than the smooth gutters seen before ablation (Fig. 7, compare $C 1, A 1)$. However, the basic layout of the AChR resembles that present before ablation. This overall pattern of AChR is retained over the next $\sim 6$ weeks

(Fig. 7C1-F1), but the fragmentation becomes even more obvious as the junction expands in size. Additionally there are small losses of receptor islands (Fig. 7D1,E1, arrows) as well as some additions of new islands (Fig. 7F1, arrowhead). As the AChR change, so does the nerve terminal. The major branches and many of the minor ones remain identifiable throughout (Fig. 7A3-F3). Some of the major branches appear to become thicker (Fig. 7, compare F3, C3), and smaller branches leading to varicosities come to hang off of them. These varicosities in the nerve terminal begin to become obvious at day 6 (Fig. 7B3) and are even more obvious by day 12 ; the terminal remains varicose thereafter. New varicosities form (Fig. 7F3, arrowhead) and these become apposed to new islands of AChR (Fig. 7F1, arrowhead). In addition, some varicosities are lost (Fig. 7D3,E3, arrows). Where the losses occur, there are losses of AChR islands and of tSC processes
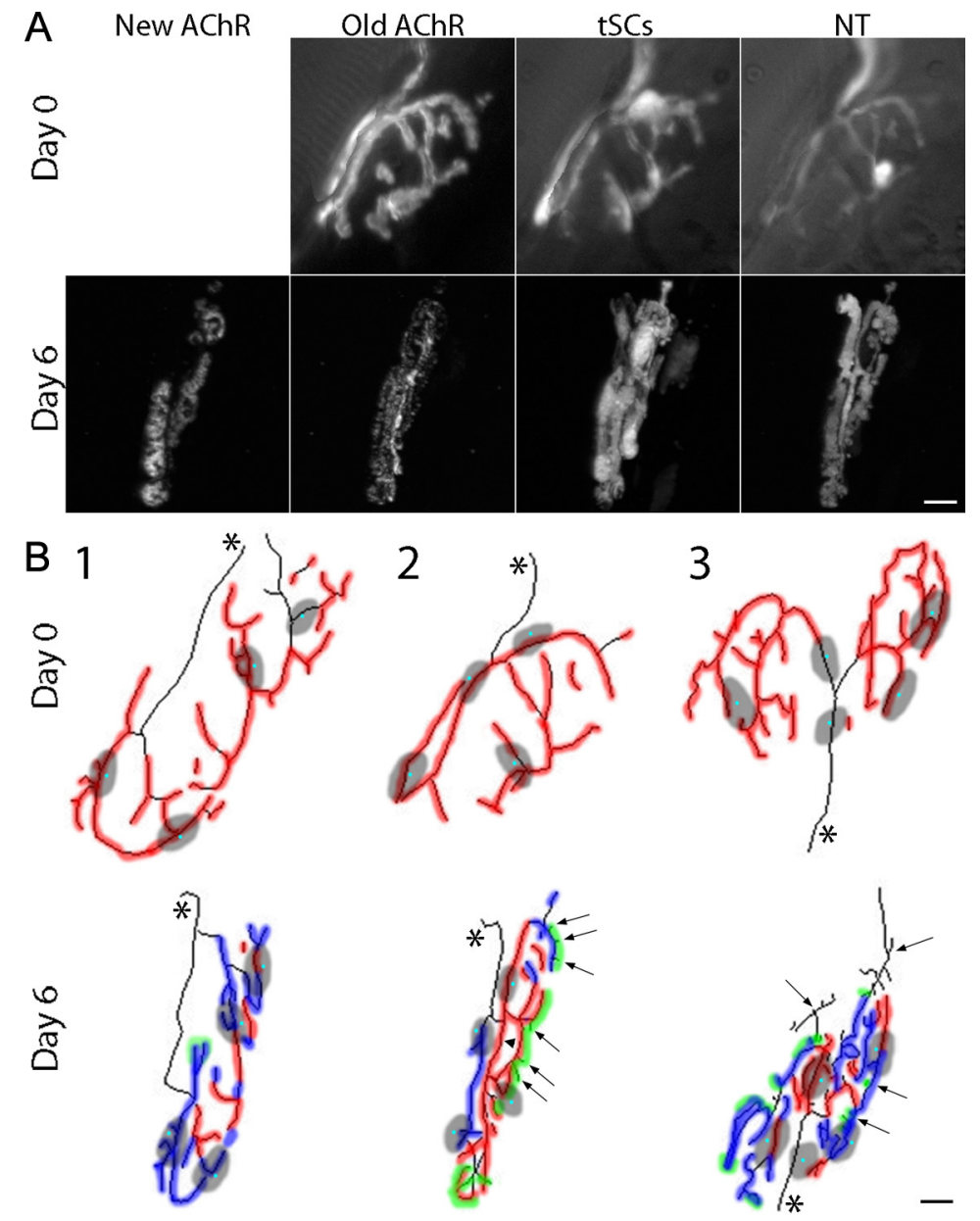

Figure 5. At $6 \mathrm{~d}$ after ablation, terminals begin to grow in association with $S$ chwann cells and establish novel synaptic sites on the regenerating muscle fiber. Vital images were collected at the time of ablation (day 0), a second-color BTX was applied at day 6 , 列 ovals with central dots, cell bodies of $\mathrm{tSC}$. Where red and green occur together, this is a site where the old and new AChRs were (indicated with small arrows), including sprouts leaving the junction site (obvious in number 3). Areas of new AChRs (green) example is in number 2 (arrowhead) where the confocals showed that the long red site in the center of the junction was located in an apparent fold above the surface of the regenerating fiber. Sprouts in number 3 grew in association with SC processes (data not shown); SCs appeared stable in number and position above each site. Scale bar: $\boldsymbol{A}, 10 \mu \mathrm{m} ; \boldsymbol{B}, 7.1 \mu \mathrm{m}$.

(Fig. 7D1,E1,D2,E2, arrows). However, because of the temporal spacing of the images, it is not possible to be certain of the precedence here. The SC number and disposition is initially unchanged by ablation (Fig. 7, compare A2, D2), although the addition of a new cell may occur on occasion.

The picture presented in Figure 7 is confirmed by observations on the additional five junctions. Most of the addition of sites of new AChR occurs between day 4 and day 12. Most new sites added after this time are small islands/fragments that do not add significantly to the total area. There is also some loss of islands and this becomes obvious only following day 12. Expansion of existing sites occurs as the fiber and junction enlarge in size; this makes it somewhat difficult to discern whether existing sites are being fragmented or whether they were already fragmented and becoming separated by the expansion. 

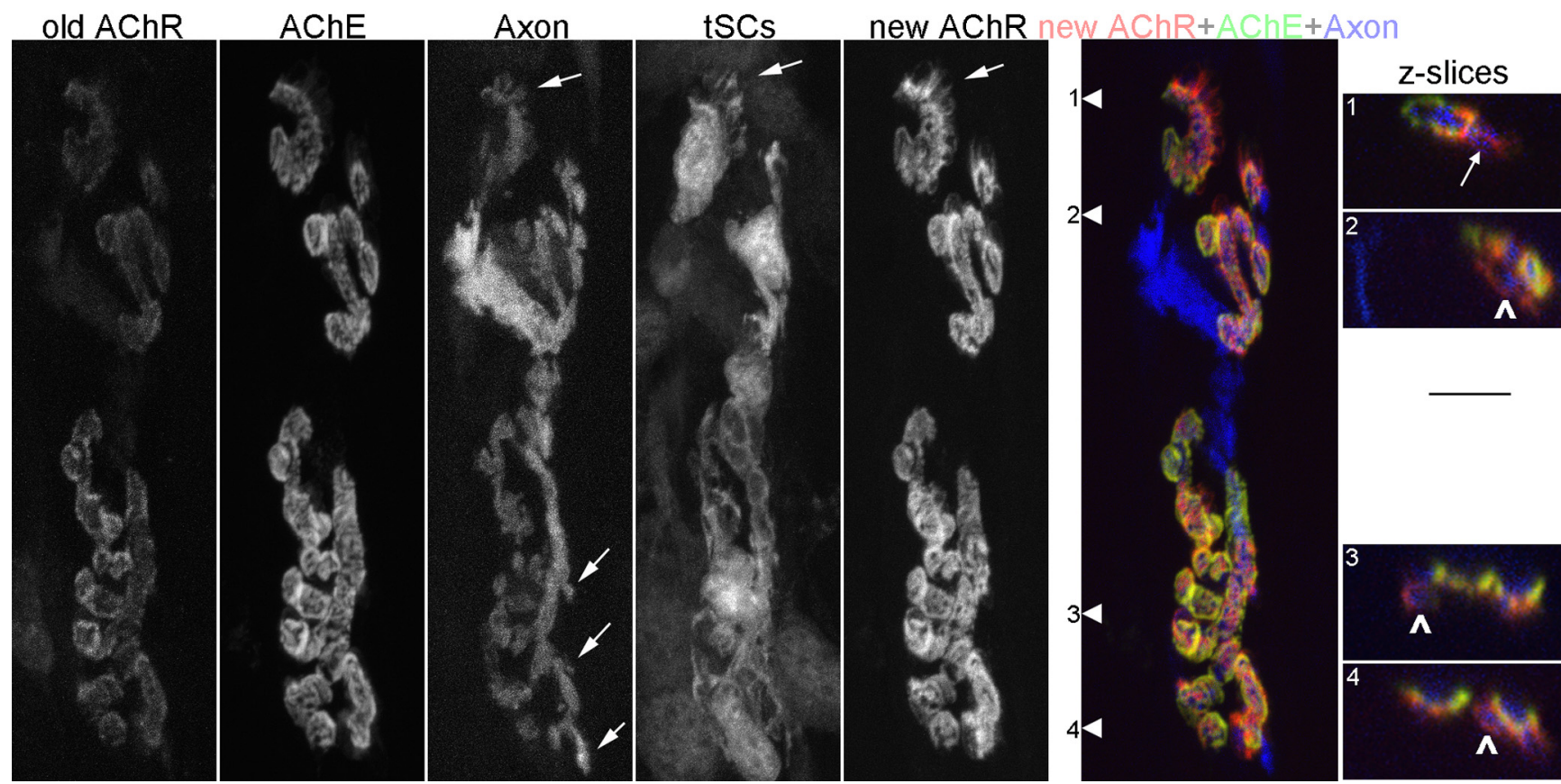

Figure 6. The nerve terminal begins to sprout short processes that contact the regenerating muscle fiber and induce novel sites of AChR both to the sides and beneath the synaptic basal lamina. Confocal views of a NMJ whose muscle fiber was ablated $6 \mathrm{~d}$ earlier. At the time of ablation, the AChRs were labeled with rho-BTX ("old AChR"). At $6 \mathrm{~d}$, after dissection and fixation, the sternomastoid was labeled with Alexa 647-conjugated fasciculin-2 for AChE and Alexa 594-BTX for "new AChR." The grayscale panels to the left show the maximum projections of the labeling for old receptor, AChE, the transgenic CFP in the axons, and the transgenic GFP in the SCS, and the new receptor, respectively. The arrows in the grayscale picture of the axon point to sprouts that extend beyond the AChE. Those at the top of the panel extend into areas where new synaptic contacts have been made (new receptors are present but no old receptors or AChE). SC extensions accompany the sprouts. The sixth panel from the left shows a colored superposition of the new AChR (red), the AChE (green), and the axon (blue). The panels to the right show z-slices through this color maximum projection; numbers to the left of the maximum projection correspond to the location of each $z$-slice. There are instances in which nerve and new AChR are to the side of the original contact outside the location of AChE (arrow in 1) and instances in which the nerve and new AChR are beneath the location of AChE (arrowheads in 2, 3, 4). DAPI-stained central nuclei were present in this fiber. The solid arrowheads in the AChE show holes/discontinuities in the labeling where in $z$-slices axons appear to enter. The results illustrated here are typical of those observed in four total junctions with the following exceptions: only two of the junctions showed the location of nerve and new receptors at planes deeper than the old receptor and AChE; one of the four junctions had a small terminal branch absent but here there were no new AChRs present. Scale bar: $10 \mu \mathrm{m}$ (all panels).

In an attempt to understand in more detail the generation of the varicosities in the nerve terminal, we returned to analyze the confocal micrographs generated in the experiments described above at various times following fiber ablation. The terminals of control junctions (i.e., those on fibers within the same muscle in which fibers were ablated) appear most commonly like smooth, but branched tubes. Varicosities, defined here as small swellings $\sim 1.5-2.0 \mu \mathrm{m}$ in diameter, are found along the course of terminal branches in control junctions, but are rare $(0-6$ per junction; $\mu=1.7 ; n=6)$. Varicosities in the terminal branches on the ablated fibers remained rare $3 \mathrm{~d}$ after ablation $(0-5$ per junction; $\mu=2.6 ; n=5)$. However, at $6 \mathrm{~d}$, by which time the fiber has regenerated, the number of varicosities had increased (4-14 per junction; $\mu=8.6 ; n=5$ ) (Fig. 5B2,B3, day 6). By $29 \mathrm{~d}$, the number encountered in a single junction was 23 . At 80 and $133 \mathrm{~d}$, there were 50-70 varicosites. They appeared most often to be varicose extensions off a larger branch of the nerve terminal. They were accompanied by small extensions of the Schwann cells. This picture suggests short processes extend from the old terminal branches and grow a short distance to regenerating fibers and upon contact with the sarcolemma of the regenerating fiber induce the appearance of new receptors.

Overall, the picture is one of a rapid formation of terminal varicosities that become apposed to small islands in the new receptors deposited in the membrane of the regenerating fiber. Some of the varicosities are dynamic, but this dynamism is most pronounced close to the time of fiber regeneration. The varicosities and the fragmented islands of receptors give rise to the strik- ing "en grappe" pattern that others have noted are typical of junctions on regenerated fibers (Couteaux et al., 1988).

Although rare, terminal Schwann cells extend processes along the regenerating fibers and guide terminal sprouts that establish new synaptic sites

As described above, during early regeneration of the muscle fiber, short nerve sprouts reach the surface of the fiber associated with processes of the tSCs. Short tSC processes were detected as early as $3 \mathrm{~d}$ after fiber damage, but not before (eight processes seen in the case of 15 junctions imaged vitally). Nerve sprouts were also associated with one-half of these eight $\mathrm{tSC}$ processes; these were approximately the same length as the SC processes. The shortness of these processes, their infrequent occurrence, and the limited resolution of the vital images do not allow conclusions about precedence. However, the frequency and length of sprouts and processes in the case of these regenerating muscles is far less than the profusion of such growth expected at early stages of nerve regeneration (Son and Thompson, 1995), suggesting a difference in the quantity or the nature of the signaling generating sprouting.

There were only a few cases in which we observed much longer nerve sprouts and SC processes. From days 4-6, in addition to the common short tSC processes found in the vicinity of the junctions, long processes were also detected beyond the synaptic site along the regenerating muscle fibers (but in only 4 of 37 junctions). In these four junctions, three had one long SC process and one had two long SC processes. Without exception, all these 
tSC extensions were occupied by terminal sprouts at some time during the vital imaging. Most of them were the same length as their associated SC processes at the time we first observed them vitally; however, no nerve sprouts were found without SCs. In one case, we saw evidence of a clear precedence. In this case, the $\mathrm{tSC}$ process was visible $5 \mathrm{~d}$ after fiber damage, but no terminal sprout was present at this time (Fig. 8C3). Then, $4 \mathrm{~d}$ later, a fine nerve sprout was found associated with the path of this SC process. When Alexa 647-BTX was applied to the muscle at this time, new AChR clusters were found to have accumulated underneath the sprout (Fig. $8 D 4)$. This observation is consistent with previous observations suggesting that SC processes guide nerve growth during fiber denervation and reinnervation (Son and Thompson, 1995).

Terminal Schwann cells also extend processes from nearby intact fibers during muscle fiber regeneration Previous studies have shown that nerve sprouting occurs from fibers adjoining a regenerating one (van Mier and Lichtman, 1994). To investigate whether this occurred in our experiments and to determine the response of SC cells on adjoining fibers, we followed some NMJs on fibers adjoining the ones we ablated. Surprisingly, tSC processes appeared on only $14 \%$ of these nearby junctions (4 of 29 junctions; data not shown). In two of these four cases, the NMJs from which the SC extensions occurred were adjacent to the regenerating fiber; in another case, the intact fiber was one fiber away, and in the last case the intact fiber was two fibers away. In all four cases, the SC processes were relatively thinner and shorter than the ones on the regenerating fibers. They also appeared $\sim 1-2 \mathrm{~d}$ later than the processes on the regenerating fibers. Two of them showed an inclination toward the regenerating fiber and the others grew along the length of their own fiber (data not shown). However, in none of these cases were any nerve sprouts associated with these SC extensions from adjoining muscle fibers. Nor were any new AChRs ever deposited along the course of these $\mathrm{SC}$ extensions. One of the $\mathrm{tSC}$ processes that emerged around day 12 after damage continued axonless for the next $8 \mathrm{~d}$; eventually (after another $10 \mathrm{~d}$ ), this process disappeared, apparently having been withdrawn (data not shown). This response of the SCs is clearly different from cases of partial denervation in which the growth of SCs and sprouts is more robust (Son and Thompson, 1995). $20 \mu \mathrm{m}$.

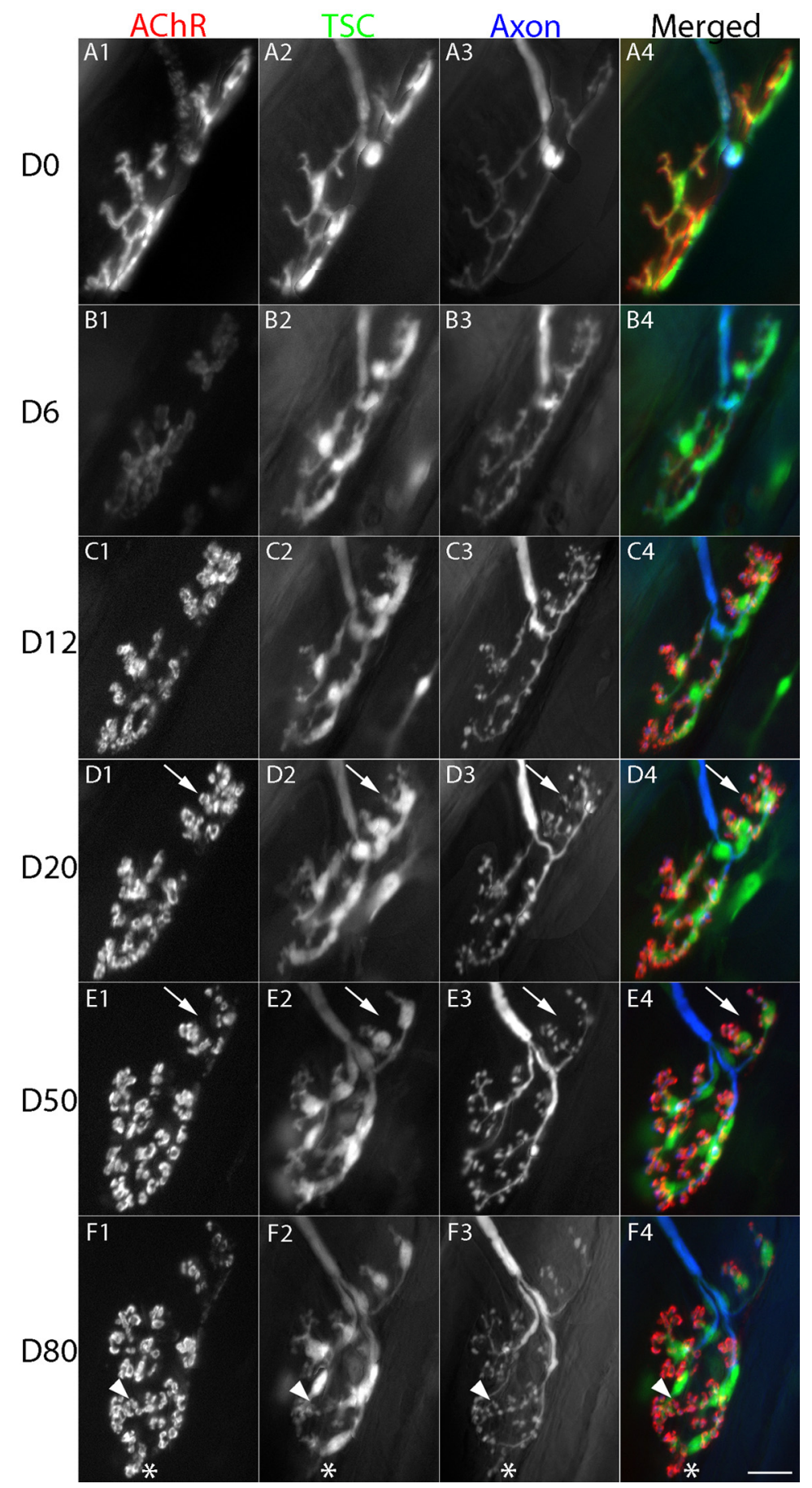

Figure 7. NMJs on the regenerated fibers undergo slow remodeling. A1-A4, Vital images of $A C h R, t S C$, and nerve terminal (NT) obtained immediately following fiber ablation (day 0). B1-F4, Images of the same components following fiber regeneration viewed over the course of $80 \mathrm{~d}$. New AChRs were labeled during imaging sessions on days $12,20,50$, and 80 with a second color of BTX and are the images presented as the $A C h R(B 1, C 1, D 1, E 1, F 1)$. In the merged images $(\boldsymbol{A 4}, \mathbf{B 4}, \mathbf{C 4}, \mathbf{D 4}, \mathbf{E 4}, \mathbf{F 4}), A C h R s$ appear red; tSCS, green; and NT, blue. Between day 20 and day 50, both tSC processes and NT lose several secondary branches (D2, D3; E2, E3, arrows). The AChR sites underneath the lost NT branches also disappear (D1, E1, arrows). Over the next $30 \mathrm{~d}$, both $\mathrm{tSC}$ processes and secondary branches of the NT are lost, and the receptors beneath those branches have become very dim. Both tSCs and NT extend new branches at two separate areas of the endplate between day 50 and day 80 and new receptor sites accumulate underneath (F1-F4, arrowheads and asterisks). AChRs become fragmented and the nerve terminal becomes varicose. Scale bar,

\section{Discussion}

Removal of the postsynaptic muscle target underneath the mouse NMJ has profound consequences for the motor nerve terminal. However, these changes do not appear immediately upon loss of 


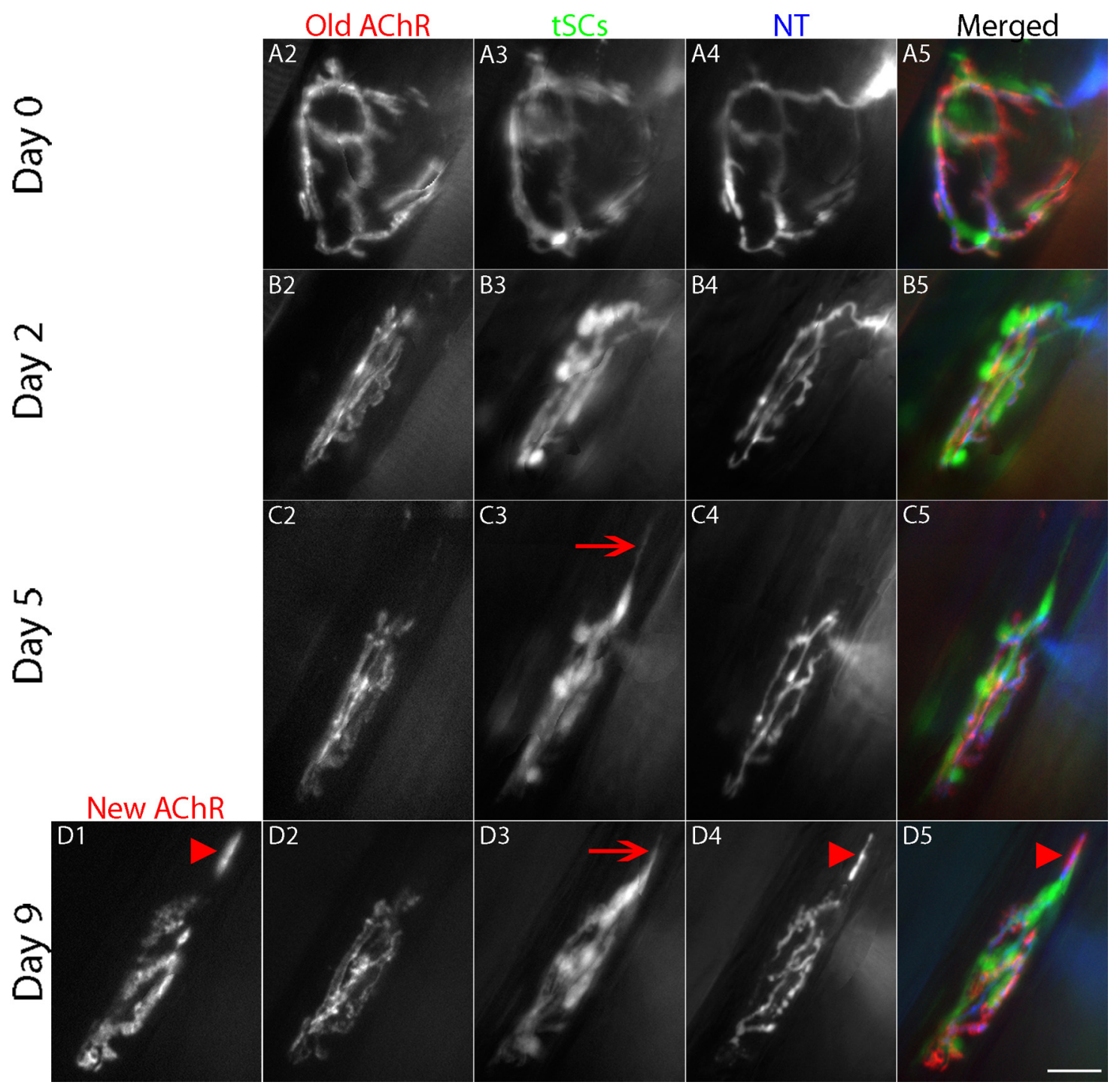

Figure 8. $\quad \mathrm{tSC}$ processes extend before nerve sprouts and these sprouts then form new synaptic sites on regenerating muscle fibers. A2-C5, D2-D5, AChR, tSCS, and nerve terminal (NT) at a synaptic site on a damaged fiber were viewed four times over the course of $9 \mathrm{~d}$. AChRs were initially labeled with rho-BTX. Images were acquired immediately following laser ablation (day 0 ), and on days 2, 5, and 9. A second color of BTX (Alexa 647) was applied on day 9 to reveal the position and the pattern of the new AChR sites. In the merged images, $A 5, B 5$, C5 are the overlays from the old AChR (red), tSCs (green), and NT (blue) acquired at the same time; D5 is the overlay from the new AChR (red), tSCs (green), and NT (blue). A long SC process was present at day 5 along the regenerating fiber ( $\mathbf{3}$, arrow), but no terminal sprout was visible at this time. However, a terminal sprout did appear $4 \mathrm{~d}$ later (D4, arrowhead) and followed the path of the preexisting $\mathbf{S C}$ process (D5, arrowhead). At the same time, new AChR had accumulated underneath the sprout (D1, D5, arrowheads). Scale bar, $20 \mu \mathrm{m}$.

this target. Other than shrinkage, as the basal lamina tube surrounding the muscle fiber collapses and folds onto itself (Marshall et al., 1977), the nerve terminal remains aligned with remnants of the old synaptic site identified by AChE and faint labeling of AChR. The branching pattern of the motor terminal, within the accuracy that can be determined from the initial images obtained vitally from the living animal, appears unchanged. This is like the situation for targetless frog motor nerve terminals (Yao, 1988; Dunaevsky and Connor, 1998). However, the terminal begins to change as the fiber regenerates. As noted previously (Rich and Lichtman, 1989a), this regeneration can be easily identified by the appearance of new binding sites for bungarotoxin in the AChRs aggregating in the membrane of the regenerating fiber. At this time it becomes clear that the new AChRs do not exactly conform to the previous synaptic site. In agreement with the previous study (Rich and Lichtman, 1989a), some portions of the site appear to gain no new receptor (obvious examples occur in Figs. 4 and 5). However, we see that almost all these sites have not lost nerve terminal branches. Rather, these branches do not come into contact with the regenerating fiber but remain positioned above its surface. Thus, the sarcolemma of the regenerating muscle fiber would not be expected to receive contactmediated signals specifying synaptic differentiation from either the old synaptic basal lamina or from the nerve terminal itself. Evidence for this as a mechanism of synaptic change comes from one particular site illustrated in the drawing of Figure 5. Here, one long terminal branch that remains in contact with the firstcolor bungarotoxin, and thus had previously been synaptic, is now above the surface of the muscle fiber and accumulates no new AChR underneath it.

We believe that the AChE labeling in our study provides compelling evidence, when combined with the fluorescent protein labeling of the nerve terminal, that the terminal remains associated with the old synaptic basal lamina and is not significantly altered in response to target loss. In contrast to other reagents that have traditionally been used to label AChE, fasciculin-2 is a small 
peptide like bungarotoxin and penetrates well into the synaptic cleft (Peng et al., 1999). Other methods of AChE labeling commonly produce a weaker staining in the depths of the synaptic gutter if a nerve terminal occupies the gutter and limits access of reagents; this weaker staining has been used to argue for occupancy of the gutter by the nerve (Letinsky et al., 1976; Wernig et al., 1980; Rich and Lichtman, 1989b). In the present study, the close apposition of the fasciculin-2 label to the nerve terminal in the $z$-stacks of the ablated fibers provides evidence of continued occupation of the synaptic basal lamina within the resolution provided by light microscopy. We cannot exclude the possibility, since our observations are based on topology of the branches, that some sites in the basal lamina have been abandoned and therefore ultimately are eliminated. However, even if such abandonment occurs, it rarely involves wholesale loss of branches, as originally reported.

That the synaptic change observed subsequent to muscle fiber regeneration is driven by relative growth of the nerve terminal and the muscle fiber is also supported by the relative positions of the old and new AChR in the plane of $z$-sections through the synapse. In many cases, new receptors are located beneath the old ones (and the old AChE). Since there are nerve sprouts reaching down to the new receptors, a simple explanation here would be that the regenerating muscle fiber, proposed to release agents inducing sprouting (van Mier and Lichtman, 1994), has caused the nerve terminal to grow very short distances through the old basal lamina toward the regenerating fiber. Such growth would produce a layer of new receptors that at least partially mirrors the layer of old receptors above it in an en face view. This is essentially what Couteaux et al. (1988) proposed on the basis of static electron microscopic observations.

The ultimate configuration of the synapses on these regenerated fibers (Fig. 7) also supports this view of how the nerve terminals grow. These terminals come to have the appearance of a planar network of main branches (most of which appear to be the original terminal branches) from which small neurites extend down to contact the muscle fiber surface. The varicose endings on many of these neurites appear to be the consequence of the growth pattern of the initial short sprouts and explain the fragmentation in the AChR pattern. Thus, the pattern of the junction would be a consequence of the nerve growing down to the fiber surface rather than growing along the fiber surface. Fragmented contacts like those seen here have been previously reported for cases of muscle fiber regeneration, including prominently the mouse model of Duchenne muscular dystrophy (Lyons and Slater, 1991) and deliberate injury to fibers with myotoxic drugs (Duchen et al., 1974; Couteaux et al., 1988) in which the form of the terminal endings has been described as en grappe (Couteaux et al., 1988). That these en grappe endings are subject to remodeling in the period immediately following fiber regeneration, whereas the synapses on other undamaged fibers in the muscle are not, is unexplained but may have to do with relative differences in how tightly varicosities versus terminals with more continuous contact adhere to the muscle fiber surface and its basal lamina.

Published studies show the remodeling of synaptic sites in rodents depends on whether innervation is present when muscle fibers regenerate (Rich and Lichtman, 1989a; Marques et al., 2005). Fibers regenerating in the absence of a nerve terminal insert new receptors that match those of the previous synaptic site; regeneration in the presence of the nerve results in differences in the deposition of new receptors relative to the old site. These observations have been interpreted as showing that inner- vation can override the signals in the original basal lamina (Rich and Lichtman, 1989a). Our results suggest that some of the differences may have a simple explanation in the degree of contact of the regenerating fiber with the old basal lamina as well as the new growth that occurs.

The SCs associated with the nerve terminals on the ablated fibers behave as the nerve terminal does, both before and after fiber regeneration. Early after fiber damage, SCs are not activated by the degeneration of the old fiber or by the invasion of immune cells. They do not proliferate or begin to grow processes, in contrast to their response to muscle denervation (Love and Thompson, 1998). An explanation of this difference in SC response in the case of denervation versus fiber degeneration may be the maintained contact with an axon when only the muscle fiber degenerates. The loss of axonal contact appears to be the major stimulus for activation of SCs. Interestingly, the SCs do begin to grow upon regeneration of the muscle fiber, extending down to the fiber surface or for short distances along the fiber surface, but only rarely lead to the formation of new synaptic sites remote from the original ones. These SC processes do not by themselves induce AChR accumulation in the muscle membrane; a nerve appears to be required. There is also very little growth of SC processes from fibers adjacent to the regenerating fiber. This suggests the sprouting signal is less intense than in the case of many denervated fibers. Interestingly, the ultrastructural studies of nerve terminals on muscle fibers degenerating in response to myotoxic drugs suggest that the nerve terminals become wrapped in Schwann cell membrane (Duchen et al., 1974; Jirmanová, 1975; Couteaux et al., 1988). At the very least, the presence of SC wrappings suggests that encounters of the regenerating muscle fiber with the old synaptic apparatus initially is with the basal lamina, at least until the SC coverings are removed or the nerve terminal penetrates through them. These wrappings may offer an explanation for the terminal varicosities that form during reestablishment of the synaptic connection.

The stability of the nerve terminal in response to removal of the target reported here stands in stark contrast to the report of rapid withdrawal of terminal processes upon injection of the postsynaptic muscle fiber with protein synthesis inhibitors that shut down synthetic activity in the subsynaptic muscle nuclei (McCann et al., 2007). How could blockade of postsynaptic protein synthesis remodel nerve terminal branches when removal of the target itself does not? Evidence suggests that factors are present at the synapse that both positively and negatively impact nerve terminal and the postsynaptic receptors. It is clear that the action of some of these factors is influenced by the release of acetylcholine from the nerve terminal and its activation of postsynaptic receptors (Balice-Gordon and Lichtman, 1994; Jennings, 1994). Indeed the activation of AChR by ACh has been shown to counter the synapse-stabilizing action of the basal lamina protein agrin (Misgeld et al., 2005). The response to ACh would be absent in the case of target ablation but not necessarily in manipulations of protein synthesis by the target.

\section{References}

Akaaboune M, Culican SM, Turney SG, Lichtman JW (1999) Rapid and reversible effects of activity on acetylcholine receptor density at the neuromuscular junction in vivo. Science 286:503-507.

Balice-Gordon RJ, Lichtman JW (1994) Long-term synapse loss induced by focal blockade of postsynaptic receptors. Nature 372:519-524.

Balice-Gordon RJ, Breedlove SM, Bernstein S, Lichtman JW (1990) Neuromuscular junctions shrink and expand as muscle fiber size is manipulated: in vivo observations in the androgen-sensitive bulbocavernosus muscle of mice. J Neurosci 10:2660-2671. 
Brooks SV, Faulkner JA (1990) Contraction-induced injury: recovery of skeletal muscles in young and old mice. Am J Physiol 258:C436-C442.

Burden SJ, Sargent PB, McMahan UJ (1979) Acetylcholine receptors in regenerating muscle accumulate at original synaptic sites in the absence of the nerve. J Cell Biol 82:412-425.

Carlson BM (1973) The regeneration of skeletal muscle. A review. Am J Anat 137:119-149.

Couteaux R, Mira JC, d'Albis A (1988) Regeneration of muscles after cardiotoxin injury. I. Cytological aspects. Biol Cell 62:171-182.

Duchen LW, Excell BJ, Patel R, Smith B (1974) Changes in motor end-plates resulting from muscle fibre necrosis and regeneration. A light and electron microscopic study of the effects of the depolarizing fraction (cardiotoxin) of Dendroaspis jamesoni venom. J Neurol Sci 21:391-417.

Dunaevsky A, Connor EA (1995) Long-term maintenance of presynaptic function in the absence of target muscle fibers. J Neurosci 15:6137-6144.

Dunaevsky A, Connor EA (1998) Stability of frog motor nerve terminals in the absence of target muscle fibers. Dev Biol 194:61-71.

Feng G, Mellor RH, Bernstein M, Keller-Peck C, Nguyen QT, Wallace M, Nerbonne JM, Lichtman JW, Sanes JR (2000) Imaging neuronal subsets in transgenic mice expressing multiple spectral variants of GFP. Neuron 28:41-51.

Gatchalian CL, Schachner M, Sanes JR (1989) Fibroblasts that proliferate near denervated synaptic sites in skeletal muscle synthesize the adhesive molecules tenascin(J1), N-CAM, fibronectin, and a heparan sulfate proteoglycan. J Cell Biol 108:1873-1890.

Harris JB, Johnson MA (1978) Further observations on the pathological responses of rat skeletal muscle to toxins isolated from the venom of the Australian tiger snake, Notechis scutatus scutatus. Clin Exp Pharmacol Physiol 5:587-600.

Jennings C (1994) Developmental neurobiology. Death of a synapse. Nature $372: 498-499$

Jirmanová I (1975) Ultrastructure of motor end-plates during pharmacologically-induced degeneration and subsequent regeneration of skeletal muscle. J Neurocytol 4:141-155.

Jones KH, Senft JA (1985) An improved method to determine cell viability by simultaneous staining with fluorescein diacetate-propidium iodide. J Histochem Cytochem 33:77-79.

Kang H, Tian L, Thompson W (2003) Terminal Schwann cells guide the reinnervation of muscle after nerve injury. J Neurocytol 32:975-985.

Kummer TT, Misgeld T, Lichtman JW, Sanes JR (2004) Nerve-independent formation of a topologically complex postsynaptic apparatus. J Cell Biol 164:1077-1087.

Le Grand F, Rudnicki MA (2007) Skeletal muscle satellite cells and adult myogenesis. Curr Opin Cell Biol 19:628-633.

Letinsky MS, Fischbeck KH, McMahan UJ (1976) Precision of reinnervation of original postsynaptic sites in frog muscle after a nerve crush. J Neurocytol 5:691-718.

Lichtman JW, Magrassi L, Purves D (1987) Visualization of neuromuscular junctions over periods of several months in living mice. J Neurosci 7:1215-1222.

Liley AW (1956) An investigation of spontaneous activity at the neuromuscular junction of the rat. J Physiol 132:650-666.

Lingle CJ, Steinbach JH (1988) Neuromuscular blocking agents. Int Anesthesiol Clin 26:288-301.

Love FM, Thompson WJ (1998) Schwann cells proliferate at rat neuromuscular junctions during development and regeneration. J Neurosci 18:9376-9385.

Lyons PR, Slater CR (1991) Structure and function of the neuromuscular junction in young adult mdx mice. J Neurocytol 20:969-981.
Magrassi L, Purves D, Lichtman JW (1987) Fluorescent probes that stain living nerve terminals. J Neurosci 7:1207-1214.

Marques MJ, Mendes ZT, Minatel E, Santo Neto H (2005) Acetylcholine receptors and nerve terminal distribution at the neuromuscular junction of long-term regenerated muscle fibers. J Neurocytol 34:387-396.

Marshall LM, Sanes JR, McMahan UJ (1977) Reinnervation of original synaptic sites on muscle fiber basement membrane after disruption of the muscle cells. Proc Natl Acad Sci U S A 74:3073-3077.

McCann CM, Nguyen QT, Santo Neto H, Lichtman JW (2007) Rapid synapse elimination after postsynaptic protein synthesis inhibition in vivo. J Neurosci 27:6064-6067.

McMahan UJ, Slater CR (1984) The influence of basal lamina on the accumulation of acetylcholine receptors at synaptic sites in regenerating muscle. J Cell Biol 98:1453-1473.

McMahan UJ, Sanes JR, Marshall LM (1978) Cholinesterase is associated with the basal lamina at the neuromuscular junction. Nature 271:172-174.

Misgeld T, Kummer TT, Lichtman JW, Sanes JR (2005) Agrin promotes synaptic differentiation by counteracting an inhibitory effect of neurotransmitter. Proc Natl Acad Sci U S A 102:11088-11093.

Papadimitriou JM, Robertson TA, Mitchell CA, Grounds MD (1990) The process of new plasmalemma formation in focally injured skeletal muscle fibers. J Struct Biol 103:124-134.

Peng HB, Xie H, Rossi SG, Rotundo RL (1999) Acetylcholinesterase clustering at the neuromuscular junction involves perlecan and dystroglycan. J Cell Biol 145:911-921.

Ribchester RR (2009) Mammalian neuromuscular junctions: modern tools to monitor synaptic form and function. Curr Opin Pharmacol 9:297-305.

Rich M, Lichtman JW (1989a) Motor nerve terminal loss from degenerating muscle fibers. Neuron 3:677-688.

Rich MM, Lichtman JW (1989b) In vivo visualization of pre- and postsynaptic changes during synapse elimination in reinnervated mouse muscle. J Neurosci 9:1781-1805.

Sanes JR, Lichtman JW (2001) Induction, assembly, maturation and maintenance of a postsynaptic apparatus. Nat Rev Neurosci 2:791-805.

Slater CR, Allen EG (1985) Acetylcholine receptor distribution on regenerating mammalian muscle fibers at sites of mature and developing nervemuscle junctions. J Physiol (Paris) 80:238-246.

Son YJ, Thompson WJ (1995) Nerve sprouting in muscle is induced and guided by processes extended by Schwann cells. Neuron 14:133-141.

Valdez G, Tapia JC, Kang H, Clemenson GD Jr, Gage FH, Lichtman JW, Sanes JR (2010) Attenuation of age-related changes in mouse neuromuscular synapses by caloric restriction and exercise. Proc Natl Acad Sci U S A 107:14863-14868.

van Mier P, Lichtman JW (1994) Regenerating muscle fibers induce directional sprouting from nearby nerve terminals: studies in living mice. J Neurosci 14:5672-5686.

Wernig A, Pécot-Dechavassine M, Stöver H (1980) Sprouting and regression of the nerve at the frog neuromuscular junction in normal conditions and after prolonged paralysis with curare. J Neurocytol 9:277-303.

Yao YM (1988) Maintenance of axon terminals at synaptic sites in the absence of muscle fibers. In: Current issues in neural regeneration research (Reier PJ, Bunge RP, Seil FJ, eds), pp 167-178. New York: Alan R. Liss.

Zuo Y, Lubischer JL, Kang H, Tian L, Mikesh M, Marks A, Scofield VL, Maika S, Newman C, Krieg P, Thompson WJ (2004) Fluorescent proteins expressed in mouse transgenic lines mark subsets of glia, neurons, macrophages, and dendritic cells for vital examination. J Neurosci 24:10999-11009. 